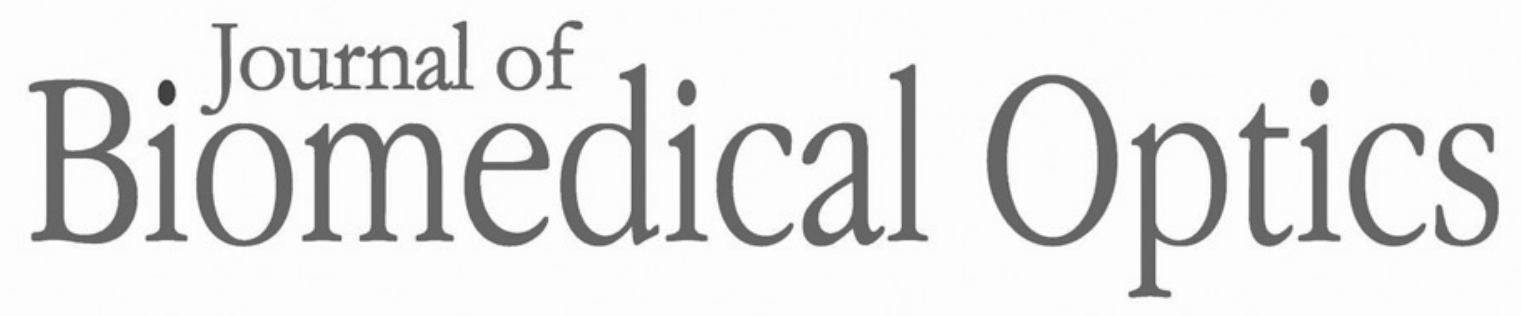

BiomedicalOptics.SPIEDigitalLibrary.org

\title{
Terahertz Biomedical Science and Technology
}

Neil T. Hunt

\section{SPIE.}




\section{Terahertz Biomedical Science and Technology}

Joo-Hiuk Son, Ed., 377 pages, ISBN: 978-1-4665-7044-3, CRC Press, Boca Raton, Florida (2014), \$149.96, hardcover.

Reviewed by Neil T. Hunt, University of Strathclyde, Department of Physics, Glasgow, United Kingdom

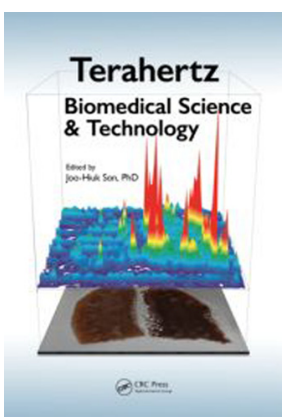

This book, edited by Joo-Huik Son, sets out to cover the science and technology that underpin the use of terahertz $(\mathrm{THz})$ spectroscopy methods for biomedical applications. The stated aims are to provide a textbook that begins by introducing terahertz waves and then takes the reader through basic $\mathrm{THz}$ spectroscopy to the application of $\mathrm{THz}$ radiation in biomedical imaging applications. This is a challenging task and accomplishing it comprehensively would entail covering a lot of ground. Having said that, the editor has assembled a large team of authors with expertise in relevant areas, and the 19 chapters do indeed manage to address important topics relevant to each of these these themes.

The book is structured into three sections: the first deals with the technology required to generate and detect $\mathrm{THz}$ waves, the second moves onto $\mathrm{THz}$ spectroscopy of biological systems, and the third to biomedical applications of $\mathrm{THz}$ radiation. Each section is a collection of chapters focusing on different topics, but there is a nice overarching theme of logical structure running through the choices of topic in each section, and indeed the book more generally. For example, the first section begins with an overview of sources and detectors, with later chapters filling in the technical details as the section progresses to the very specific topic of waveguides for endoscopy. This pattern is maintained in subsequent sections, with the second section relating to fundamental studies of biological systems beginning, logically, with work on water and then moving through proteins and amino acids to consider the effects of $\mathrm{THz}$ waves on biological matter.

The individual chapters of the book are written by different authors or teams of authors, and so the depth of coverage and writing styles do vary. However, all authors are experts in their chosen topic and the combination of broader introductions with higher-level more detailed reviews of the frontiers of specific research areas works well and means that the book will provide an accessible resource for readers of all levels. I can well imagine this text being used as a starting point for someone looking to start out in $\mathrm{THz}$ methods, such as a new $\mathrm{PhD}$ student. Furthermore, progression to the more focused chapters later in the text will allow the student to advance as they go through the text, while also providing interest for more seasoned researchers in the field.

In summary, this book sets out with the challenge of covering a broad and richly varied topic. This prohibits a completely comprehensive review, but what has been achieved provides a nice starting point from which one can delve more deeply into the research areas covered. 